Mohammad Hayal Alotaibi, Hanan A. Mohamed, Bakr F. Abdel-Wahab, Amany S. Hegazy, Benson M. Kariuki* and Gamal A. El-Hiti*

\title{
Crystal structure of $\boldsymbol{N}^{\prime}$-(1-(2-hydroxyphenyl) ethylidene)-5-methyl-1-phenyl-1H-1,2,3-triazole- 4-carbohydrazide, $\mathrm{C}_{18} \mathrm{H}_{17} \mathrm{~N}_{5} \mathrm{O}_{2}$
}

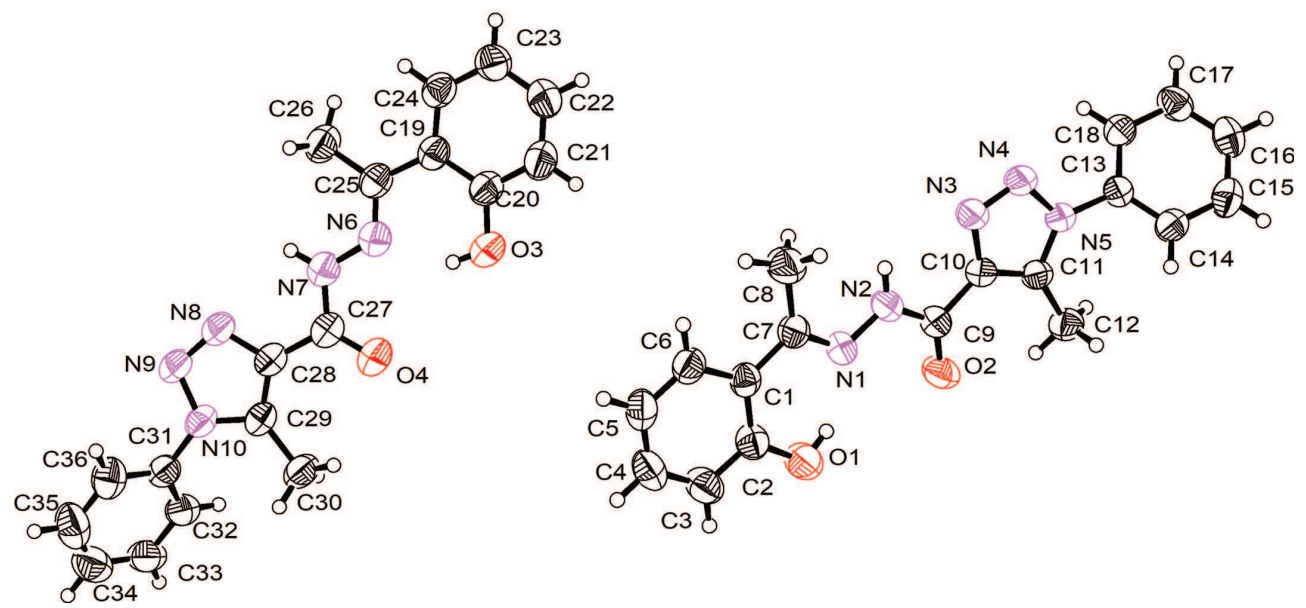

https://doi.org/10.1515/ncrs-2018-0417

Received October 8, 2018; accepted October 26, 2018; available online November 13, 2018

\section{Abstract \\ $\mathrm{C}_{18} \mathrm{H}_{17} \mathrm{~N}_{5} \mathrm{O}_{2}$, monoclinic, $P 2_{1} / n$ (no. 14), $a=20.3702(7) \AA$, $b=7.3482(2) \AA, \quad c=23.2504(10) \AA, \quad \beta=106.507(4)^{\circ}$, $V=3336.8(2) \AA^{3}, Z=8, R_{\mathrm{gt}}(F)=0.0571, w R_{\text {ref }}\left(F^{2}\right)=0.1507$, $T=296(2) \mathrm{K}$.}

CCDC no.: 1875460

\footnotetext{
*Corresponding authors: Benson M. Kariuki, School of Chemistry, Cardiff University, Main Building, Park Place, Cardiff CF10 3AT, UK, e-mail: kariukib@cardiff.ac.uk; and Gamal A. El-Hiti, Department of Optometry, College of Applied Medical Sciences, King Saud University, P.O. Box 10219, Riyadh 11433, Saudi Arabia, e-mail: gelhiti@ksu.edu.sa

Mohammad Hayal Alotaibi: National Center for Petrochemicals Technology, King Abdulaziz City for Science and Technology, P.0. Box 6086, Riyadh 11442, Saudi Arabia

Hanan A. Mohamed and Bakr F. Abdel-Wahab: Department of Chemistry, College of Science and Humanities, Shaqra University, Duwadimi, Saudi Arabia; and Applied Organic Chemistry Department, National Research Centre, Dokki, Giza, Egypt Amany S. Hegazy: School of Chemistry, Cardiff University, Main Building, Park Place, Cardiff CF10 3AT, UK
}

Table 1: Data collection and handling.

\begin{tabular}{ll}
\hline Crystal: & Colourless block \\
Size: & $0.37 \times 0.20 \times 0.15 \mathrm{~mm}$ \\
Wavelength: & Mo $K \alpha$ radiation $(0.71073 \AA)$ \\
$\mu:$ & $0.09 \mathrm{~mm}^{-1}$ \\
Diffractometer, scan mode: & SuperNova, $\omega$ \\
$\theta_{\max }$, completeness: & $29.7^{\circ},>99 \%$ \\
$N\left(h k l_{\text {measured }}, N\left(h k l l_{\text {unique }}, R_{\text {int }}:\right.\right.$ & $32673,8345,0.033$ \\
Criterion for $I_{\text {obs }}, N(h k)_{\text {gt }}:$ & $I_{\text {obs }}>2 \sigma\left(I_{\text {obs }}\right), 5134$ \\
$N(\text { param })_{\text {refined }}:$ & 458 \\
Programs: & CrysAlis \\
& WinGX $[1]$, SHELX $[2,3]$, \\
&
\end{tabular}

The asymmetric unit, containing two crystallographically independent molecules, of the title crystal structure is shown in the figure. Tables 1 and 2 contain details on crystal structure and measurement conditions and a list of the atoms including atomic coordinates and displacement parameters.

\section{Source of material}

The title compound was synthesized based on a literature procedure [5] and recrystallized from dimethylformamide to give colourless block crystals (86\%).

\section{Experimental details}

All hydrogen atoms were placed in calculated positions and refined using a riding model. $\mathrm{N}-\mathrm{H}$ bonds were fixed at 
Table 2: Fractional atomic coordinates and isotropic or equivalent isotropic displacement parameters $\left(\AA^{2}\right)$.

\begin{tabular}{|c|c|c|c|c|}
\hline Atom & $x$ & $y$ & $z$ & $\boldsymbol{U}_{\text {iso }}{ }^{*} / \boldsymbol{U}_{\mathrm{eq}}$ \\
\hline $\mathrm{C} 1$ & $0.12057(9)$ & $0.8874(2)$ & $0.47514(9)$ & $0.0489(4)$ \\
\hline $\mathrm{C} 2$ & $0.08609(10)$ & $0.8835(3)$ & $0.41350(9)$ & $0.0565(5)$ \\
\hline C3 & $0.12032(12)$ & $0.9311(3)$ & $0.37181(10)$ & $0.0689(6)$ \\
\hline H3 & 0.097213 & 0.927515 & 0.331097 & $0.083^{*}$ \\
\hline $\mathrm{C} 4$ & $0.18760(12)$ & $0.9833(3)$ & $0.38990(11)$ & $0.0691(6)$ \\
\hline $\mathrm{H} 4$ & 0.210018 & 1.013592 & 0.361514 & $0.083^{*}$ \\
\hline $\mathrm{C} 5$ & $0.22207(10)$ & $0.9909(3)$ & $0.44999(11)$ & $0.0644(6)$ \\
\hline H5 & 0.267610 & 1.027692 & 0.462396 & $0.077^{\star}$ \\
\hline C6 & $0.18894(10)$ & $0.9440(3)$ & $0.49135(10)$ & $0.0582(5)$ \\
\hline H6 & 0.212782 & 0.950030 & 0.531862 & $0.070^{*}$ \\
\hline $\mathrm{C7}$ & $0.08730(9)$ & $0.8324(3)$ & $0.52096(9)$ & $0.0509(4)$ \\
\hline $\mathrm{C} 8$ & $0.12478(12)$ & $0.8414(4)$ & $0.58642(10)$ & $0.0779(7)$ \\
\hline $\mathrm{H} 8 \mathrm{~A}$ & 0.102238 & 0.926203 & 0.605873 & $0.117^{\star}$ \\
\hline H8B & 0.171001 & 0.880668 & 0.591325 & $0.117^{*}$ \\
\hline $\mathrm{H} 8 \mathrm{C}$ & 0.125129 & 0.723117 & 0.604032 & $0.117^{\star}$ \\
\hline $\mathrm{C} 9$ & $-0.07306(10)$ & $0.6544(3)$ & $0.52256(9)$ & $0.0519(5)$ \\
\hline C10 & $-0.10264(9)$ & $0.6101(3)$ & $0.57162(8)$ & $0.0480(4)$ \\
\hline C11 & $-0.16850(9)$ & $0.5589(2)$ & $0.56824(8)$ & $0.0466(4)$ \\
\hline C12 & $-0.22810(10)$ & $0.5189(3)$ & $0.51571(9)$ & $0.0580(5)$ \\
\hline $\mathrm{H} 12 \mathrm{~A}$ & -0.252997 & 0.417098 & 0.524717 & $0.087^{\star}$ \\
\hline $\mathrm{H} 12 \mathrm{~B}$ & -0.212234 & 0.490555 & 0.481621 & $0.087^{*}$ \\
\hline $\mathrm{H} 12 \mathrm{C}$ & -0.257561 & 0.623413 & 0.506853 & $0.087^{\star}$ \\
\hline C13 & $-0.22141(9)$ & $0.5069(2)$ & $0.65369(8)$ & $0.0466(4)$ \\
\hline C14 & $-0.28706(9)$ & $0.5689(3)$ & $0.62847(9)$ & $0.0563(5)$ \\
\hline H14 & -0.297984 & 0.632300 & 0.592378 & $0.068^{\star}$ \\
\hline C15 & $-0.33675(10)$ & $0.5359(3)$ & $0.65739(11)$ & $0.0658(6)$ \\
\hline H15 & -0.381453 & 0.575041 & 0.640188 & 0.079 * \\
\hline C16 & $-0.32030(11)$ & $0.4458(3)$ & $0.71119(11)$ & $0.0650(6)$ \\
\hline H16 & -0.353582 & 0.426215 & 0.730834 & $0.078^{*}$ \\
\hline C17 & $-0.25458(11)$ & $0.3844(3)$ & $0.73618(10)$ & $0.0611(5)$ \\
\hline H17 & -0.243494 & 0.323191 & 0.772692 & $0.073^{\star}$ \\
\hline C18 & $-0.20502(10)$ & $0.4134(3)$ & $0.70715(9)$ & $0.0554(5)$ \\
\hline H18 & -0.160779 & 0.369865 & 0.723658 & $0.066^{*}$ \\
\hline C19 & $0.49738(9)$ & $0.2062(3)$ & $0.61744(10)$ & $0.0540(5)$ \\
\hline $\mathrm{C} 20$ & $0.42966(10)$ & $0.2652(3)$ & $0.59025(10)$ & $0.0621(5)$ \\
\hline $\mathrm{C} 21$ & $0.38566(11)$ & $0.2931(3)$ & $0.62507(12)$ & $0.0749(7)$ \\
\hline $\mathrm{H} 21$ & 0.340885 & 0.329834 & 0.606804 & $0.090^{*}$ \\
\hline $\mathrm{C} 22$ & $0.40661(12)$ & $0.2679(3)$ & $0.68561(12)$ & $0.0724(6)$ \\
\hline $\mathrm{H} 22$ & 0.376246 & 0.288004 & 0.708201 & $0.087^{\star}$ \\
\hline $\mathrm{C} 23$ & $0.47271(11)$ & $0.2125(3)$ & $0.71350(11)$ & $0.0678(6)$ \\
\hline $\mathrm{H} 23$ & 0.487254 & 0.195850 & 0.754838 & $0.081^{*}$ \\
\hline C24 & $0.51682(10)$ & $0.1823(3)$ & $0.67939(10)$ & $0.0607(5)$ \\
\hline $\mathrm{H} 24$ & 0.561273 & 0.144453 & 0.698396 & $0.073^{*}$ \\
\hline $\mathrm{C} 25$ & $0.54578(10)$ & $0.1731(3)$ & $0.58201(10)$ & $0.0573(5)$ \\
\hline $\mathrm{C} 26$ & $0.61401(10)$ & $0.0825(3)$ & $0.60817(11)$ & $0.0732(6)$ \\
\hline $\mathrm{H} 26 \mathrm{~A}$ & 0.617815 & -0.021000 & 0.584101 & $0.110^{*}$ \\
\hline $\mathrm{H} 26 \mathrm{~B}$ & 0.617676 & 0.043331 & 0.648354 & $0.110^{*}$ \\
\hline $\mathrm{H} 26 \mathrm{C}$ & 0.650059 & 0.167180 & 0.608726 & $0.110^{*}$ \\
\hline $\mathrm{C} 27$ & $0.53785(10)$ & $0.2418(3)$ & $0.43069(10)$ & $0.0608(5)$ \\
\hline $\mathrm{C} 28$ & $0.58545(10)$ & $0.2273(3)$ & $0.39434(9)$ & $0.0567(5)$ \\
\hline C29 & $0.57247(9)$ & $0.2507(3)$ & $0.33380(9)$ & $0.0540(5)$ \\
\hline C30 & $0.50873(10)$ & $0.3001(3)$ & $0.28653(10)$ & $0.0689(6)$ \\
\hline $\mathrm{H} 30 \mathrm{~A}$ & 0.520297 & 0.367360 & 0.255371 & $0.103^{*}$ \\
\hline Н30В & 0.480210 & 0.373509 & 0.303775 & $0.103^{*}$ \\
\hline $\mathrm{H} 30 \mathrm{C}$ & 0.484577 & 0.191345 & 0.269959 & $0.103^{*}$ \\
\hline C31 & $0.65374(10)$ & $0.2345(3)$ & $0.26991(10)$ & $0.0561(5)$ \\
\hline
\end{tabular}

Table 2 (continued)

\begin{tabular}{lrrrr}
\hline Atom & $\boldsymbol{x}$ & $\boldsymbol{y}$ & $\boldsymbol{z}$ & $\boldsymbol{U}_{\text {iso }} / \boldsymbol{U}_{\text {eq }}$ \\
\hline C32 & $0.61490(11)$ & $0.1478(3)$ & $0.21909(10)$ & $0.0640(5)$ \\
H32 & 0.575629 & 0.084315 & 0.219738 & $0.077^{*}$ \\
C33 & $0.63476(13)$ & $0.1562(3)$ & $0.16747(11)$ & $0.0741(6)$ \\
H33 & 0.608371 & 0.099877 & 0.132765 & $0.089^{*}$ \\
C34 & $0.69322(15)$ & $0.2471(4)$ & $0.16680(13)$ & $0.0838(8)$ \\
H34 & 0.706720 & 0.250872 & 0.131821 & $0.101^{*}$ \\
C35 & $0.73196(14)$ & $0.3326(4)$ & $0.21768(15)$ & $0.0876(8)$ \\
H35 & 0.771589 & 0.394283 & 0.216972 & $0.105^{*}$ \\
C36 & $0.71239(11)$ & $0.3275(3)$ & $0.27001(12)$ & $0.0734(6)$ \\
H36 & 0.738326 & 0.385813 & 0.304504 & $0.088^{*}$ \\
N1 & $0.02520(8)$ & $0.7749(2)$ & $0.50145(7)$ & $0.0536(4)$ \\
N2 & $-0.00829(8)$ & $0.7204(2)$ & $0.54201(7)$ & $0.0564(4)$ \\
H2 & 0.011791 & 0.728044 & 0.579840 & $0.068^{*}$ \\
N3 & $-0.06542(8)$ & $0.6244(3)$ & $0.63013(7)$ & $0.0591(4)$ \\
N4 & $-0.10436(8)$ & $0.5859(3)$ & $0.66399(7)$ & $0.0599(4)$ \\
N5 & $-0.16782(7)$ & $0.5458(2)$ & $0.62651(7)$ & $0.0476(4)$ \\
N6 & $0.52485(9)$ & $0.2239(2)$ & $0.52699(9)$ & $0.0629(5)$ \\
N7 & $0.56550(9)$ & $0.2020(3)$ & $0.48976(8)$ & $0.0663(5)$ \\
H7 & 0.607043 & 0.164449 & 0.503194 & $0.080^{*}$ \\
N8 & $0.65296(8)$ & $0.1891(3)$ & $0.41904(8)$ & $0.0667(5)$ \\
N9 & $0.68330(8)$ & $0.1874(3)$ & $0.37689(8)$ & $0.0685(5)$ \\
N10 & $0.63434(8)$ & $0.2248(2)$ & $0.32432(8)$ & $0.0570(4)$ \\
01 & $0.01960(8)$ & $0.8355(3)$ & $0.39170(7)$ & $0.0796(5)$ \\
H1 & 0.005693 & 0.795392 & 0.419006 & $0.119^{*}$ \\
O2 & $-0.10394(7)$ & $0.6361(2)$ & $0.46992(6)$ & $0.0719(4)$ \\
03 & $0.40414(8)$ & $0.2961(3)$ & $0.53053(8)$ & $0.0874(5)$ \\
H3A & 0.435081 & 0.288670 & 0.514508 & $0.131^{*}$ \\
O4 & $0.47790(8)$ & $0.2840(3)$ & $0.41023(8)$ & $0.0838(5)$ \\
\hline & & & &
\end{tabular}

$0.86 \AA$ (AFIX 43 instruction in SHELXL [4]), with displacement parameters 1.2 times $U_{\text {eq }}(\mathrm{N})$. Aromatic $\mathrm{C}-\mathrm{H}$ distances were set to $0.93 \AA$ (AFIX 43) and their $U_{\text {iso }}(\mathrm{H})$ set to 1.2 times the $U_{\text {eq }}(\mathrm{C})$. Methyl C-H distances were set to $0.96 \AA$ and their $U_{\text {iso }}(\mathrm{H})$ to 1.5 times the $U_{\text {eq }}(\mathrm{C})$ with the groups allowed to rotate about the $\mathrm{C}-\mathrm{C}$ bonds (AFIX 137). $\mathrm{O}-\mathrm{H}$ bonds were fixed at $0.82 \AA$ (AFIX 147), with displacement parameters 1.2 times $U_{\text {eq }}(0)$.

\section{Comment}

Various 1H-1,2,3-triazoles show miscellaneous biological activities [6-10]. Recently, we have reported the crystal structures for two 1H-1,2,3-triazole-4-carbohydrazides [11, 12].

The asymmetric unit consists of two crystallographically independent molecules ( $c f$. the figure). Bond lengths and angles excellently fit with those of our previous experiments [11, 12]. The twist angles between the planes through the hydroxyphenyl and the triazolyl groups are $3.37(6)^{\circ}$ for molecule (C1-C18, A) and 6.02(6) ${ }^{\circ}$ for the molecule (C19-C36, B). The angles between the planes through the triazolyl and phenyl groups are $37.05(6)^{\circ}$ for $\mathbf{A}$ and $46.14(6)^{\circ}$ for $\mathbf{B}$.

The molecules are stacked along [010] in the crystal structure with A and B forming separate columns. 
Interactions of $\pi-\pi$ type occur between hydroxyphenyl and triazolyl groups of neighbouring molecules related by inversion symmetry within the columns. The associated centroidto-centroid distances are $3.97 \AA$ and $3.66 \AA$ for molecules A and $\mathbf{B}$ respectively. Intramolecular $\mathrm{O}-\mathrm{H} \cdots \mathrm{N}$ hydrogen bonds are observed with $\mathrm{O} \cdots \mathrm{N}$ distances of 2.561(2) $\AA$ and 2.540(2) $\AA$ and $\mathrm{O}-\mathrm{H} \cdots \mathrm{N}$ angles of $144.3^{\circ}$ and $143.8^{\circ}$ for $\mathbf{A}$ and $\mathrm{B}$ respectively.

Acknowledgements: Mohammad Hayal Alotaibi thanks King Abdulaziz City for Science and Technology (KACST), Saudi Arabia for financial support (Award No. 020-0180).

\section{References}

1. Rigaku Oxford Diffraction: CrysAlis ${ }^{\mathrm{PRO}}$. Rigaku Oxford Diffraction, Yarnton, England (2015).

2. Sheldrick, G. M.: A short history of SHELX. Acta Crystallogr. A64 (2008) 112-122.

3. Sheldrick, G. M.: Crystal structure refinement with SHELXL. Acta Crystallogr. C71 (2015) 3-8.

4. Farrugia, L. J.: WinGX and ORTEP for Windows: an update. J. Appl. Crystallogr. 45 (2012) 849-854.

5. Youssef, N. S.; El-Zahany, E. A. M.; Drweesh, S. A.; AbdelWahab, B. F.; Ali, M. M.: Synthesis, characterization and anticancer activity of some transition metal complexes of new Schiff base triazole derivatives. Smart Nanocomposites 5 (2014) 89-109.
6. Ferreira, S. B.; Sodero, A. C.; Cardoso, M. F.; Lima, E. S.; Kaiser, C. R.; Silva, F. P.; Ferreira, V. F.: Synthesis, biological activity, and molecular modeling studies of $1 \mathrm{H}$-1,2,3-triazole derivatives of carbohydrates as alpha-glucosidases inhibitors. J. Med. Chem. 53 (2010) 2364-2375.

7. Pokhodylo, N.; Shyyka, O.; Matiychuk, V.: Synthesis of 1,2,3triazole derivatives and evaluation of their anticancer activity. Sci. Pharm. 81 (2013) 663-676.

8. Reddy, P. V. B.; Kamala Prasad, V.; Manjunath, G.; Venkata Ramana, P. V.: Synthesis, characterization and evaluation of antibacterial activity of $(E)-N$-(substituted benzylidene)2-(2-fluorobenzyl)-5-ethyl-2H-1,2,3-triazole-4-carbohydrazides. Ann. Pharm. Fr. 74 (2016) 350-357.

9. Sadeghpour, H.; Khabnadideh, S.; Zomorodian, K.; Pakshir, K.; Hoseinpour, K.; Javid, N.; Faghih-Mirzaei, E.; Rezaei, Z.: Design, synthesis, and biological activity of new triazole and nitrotriazole derivatives as antifungal agents. Molecules 22 (2017) 1150.

10. Slámová, K.; Marhol, P.; Bezouska, K.; Lindkvist, L.; Hansen, S. G.; Kren, V.; Jensen, H. H.: Synthesis and biological activity of glycosyl-1H-1,2,3-triazoles. Bioorg. Med. Chem. Lett. 20 (2010) 4263-4265.

11. Geesi, M. H.; Mohamed, H. A.; Abdel-Wahab, B. F.; Hegazy, A. S.; Kariuki, B. M.; El-Hiti, G. A.: Crystal structure of ethyl 4-amino-5-(5-methyl-1-(4-tolyl)-1H-1,2,3-triazole4-carbonyl)-2-(phenylamino)thiophene-3-carboxylate, $\mathrm{C}_{24} \mathrm{H}_{23} \mathrm{~N}_{5} \mathrm{O}_{3}$ S. Z. Kristallogr. NCS 233 (2018) 673-674.

12. El-Hiti, G. A.; Abdel-Wahab, B. F.; Alotaibi, M. H.; Yousif, E.; Hegazy, A. S.; Kariuki, B. M.: 5-Methyl-1-(4-methylphenyl)- $N^{\prime}$-[1(1H-pyrrol-2-yl)ethylidene]-1H-1,2,3-triazole-4-carbohydrazide monohydrate. IUCrData 3 (2018) x181162. 